\title{
A secondary resonance in Mercury's rotation
}

\author{
Sandrine D'Hoedt • Benoît Noyelles · Julien Dufey • \\ Anne Lemaître
}

Received: 20 October 2009 / Revised: 12 February 2010 / Accepted: 16 February 2010 /

Published online: 13 March 2010

(C) Springer Science+Business Media B.V. 2010

\begin{abstract}
The resonant rotation of Mercury can be modelised by a kernel model on which we can add perturbations. Our kernel model is a two-degree of freedom one written in Hamiltonian formalism. For this kernel, we consider that Mercury is solid and rotates on a Keplerian orbit. By introducing the perturbations due to the other planets of the Solar System, it appears that, in a particular case, our slow degree of freedom may enter into a 1:1 resonance with the Great Inequality of Jupiter and Saturn. Actually, as the moments of inertia of Mercury are still poorly known, this phenomenon cannot be excluded.
\end{abstract}

Keywords Mercury $\cdot$ Planetary rotation $\cdot$ Hamiltonian formalism $\cdot$ Resonance · Great Inequality $\cdot$ Kernel models

\section{Introduction}

The missions MESSENGER and BepiColombo being real catalysers for the research on Mercury, more and more precise studies and theories emerge until a few years. Let us cite a few of the most recent ones: Margot (2009), who presents revised values of the north pole orientation, Dufey et al. (2008), who model the planetary perturbations on Mercurys libration in longitude and D'Hoedt and Lemaitre (2008), who show that Mercury stays in the Cassini forced state in an adiabatic way under the action of planetary long periodic terms.

However, shorter planetary periodic terms can, in some cases, induce a secondary resonance, it is for instance the case of Titan Noyelles (2008). To model this secondary resonance due to the influence of other planets of the Solar System in Mercury's rotation, we have to add the planetary perturbations to our kernel model. Our kernel model (D'Hoedt and Lemaitre 2004) is a two-degree of freedom one written in Hamiltonian formalism. Moreover we make

S. D’Hoedt $(\varangle) \cdot$ B. Noyelles $\cdot$ J. Dufey $\cdot$ A. Lemaître FUNDP, rempart de la Vierge, 8, 5000 Namur, Belgium e-mail: sandrine.dhoedt@fundp.ac.be

B. Noyelles

IMCCE_Paris Observatory / UPMC / USTL, 77 avenue Denfert-Rochereau, 75014 Paris, France 
the following hypothesis: Mercury is a solid body, its orbit is Keplerian orbit, it rotates around its axis of greatest inertia and no dissipative forces acts on its movement. If we compare the free precession period of our model with the one of the Great Inequality of Jupiter and Saturn, they are clearly of the same order of magnitude (respectively 1,065.08 and 883.28 years). However, the 1,065.08 period computed from our model was based on the hypotheses that Mercury is a rigid body and if we introduce a liquid core as, e.g., Dufey et al. (2009); Peale (1976); Peale et al. (2008, 2009), we may obtain the exact equality between the two periods for a peculiar value of $C_{m} / C$ where $C$ is the polar moment of inertia of Mercury and $C_{m}$ the one of its mantle. This assumption, even though theoretical, must not be excluded because this latest ratio is still poorly known. The missions Messenger and BepiColombo should give us a better knowledge of it (Milani et al. 2001).

In Sect. 2, we give a summary of our kernel rigid model and of the main results (equilibrium values, proper periods) obtained from it.

In Sect. 3, we consider that Mercury has a liquid core with the value of $C_{m} / C$ needed to have a 1:1 resonance with the Great Inequality of Jupiter and Saturn that we introduce in the orbital elements thanks to Simon's series at first order.

In Sect. 4, we define the secondary resonant angle from the action-angle coordinates and we compute its proper period.

In Sect. 5, we analyse the influence of the secondary resonance on the main degrees of freedom.

\section{Summary of the kernel model}

In this model, we consider Mercury as a rigid body, we do not take into account the planetary perturbations and we assume that the Spin axis and the axis of greatest inertia are aligned. In this way, our two-degree of freedom Hamiltonian averaged on the mean anomaly can be written as follows (see D’Hoedt and Lemaître (2004) for details):

$$
\begin{aligned}
\langle\mathcal{H}\rangle= & -\frac{m^{3} \mu^{2}}{2\left(\Lambda_{o}-\frac{3 \Lambda_{1}}{2}\right)^{2}}+\frac{\Lambda_{1}^{2}}{2 C}-\frac{\mathcal{G} M m^{7} \mu^{3} R_{e}^{2}}{\left(\Lambda_{0}-\frac{3}{2} \Lambda_{1}\right)^{6}} \\
& \times\left[\frac { 1 } { 2 } C _ { 2 } ^ { 0 } ( 1 + \frac { 3 e ^ { 2 } } { 2 } ) \left(-\frac{1}{4}\left(-1+3 \cos i_{o}^{2}\right)\left(-1+3 \cos ^{2} K\right)\right.\right. \\
& -3 \cos i_{o} \cos K \cos \left(\sigma_{3}\right) \sin i_{o} \sin K \\
& \left.-\frac{3}{4}\left(1-\cos ^{2} i_{o}\right)\left(1-\cos ^{2} K\right) \cos \left(2 \sigma_{3}\right)\right) \\
& +3 C_{2}^{2}\left(\frac{7 e}{2}-\frac{123 e^{3}}{16}\right)\left(\frac{1}{16}\left(1-\cos i_{o}\right)^{2}(1-\cos K)^{2} \cos \left(2 \sigma_{1}+4 \sigma_{3}\right)\right. \\
& +\frac{1}{4}\left(1-\cos i_{o}\right)(1-\cos K) \sin i_{o} \sin K \cos \left(2 \sigma_{1}+3 \sigma_{3}\right) \\
& +\frac{3}{8} \sin ^{2} i_{o} \sin ^{2} K \cos \left(2 \sigma_{1}+2 \sigma_{3}\right) \\
& +\frac{1}{4}\left(1+\cos i_{o}\right)(1+\cos K) \sin i_{o} \sin K \cos \left(2 \sigma_{1}+\sigma_{3}\right) \\
& \left.\left.+\frac{1}{16}\left(1+\cos i_{o}\right)^{2}(1+\cos K)^{2} \cos \left(2 \sigma_{1}\right)\right)\right]
\end{aligned}
$$


Fig. 1 The ecliptic frame $\left(\mathbf{X}_{\mathbf{0}}, \mathbf{Y}_{\mathbf{0}}, \mathbf{Z}_{\mathbf{0}}\right)$ with $\mathbf{X}_{\mathbf{0}}$ and $\mathbf{Y}_{\mathbf{0}}$ fixed in the ecliptic plane at a determined epoch and $\mathbf{Z}_{\mathbf{0}}$ normal to the ecliptic plane and the body frame $\left(\mathbf{X}_{\mathbf{3}}, \mathbf{Y}_{\mathbf{3}}, \mathbf{Z}_{\mathbf{3}}\right)$ with $\mathbf{X}_{\mathbf{3}}$ in the direction of the axis of biggest inertia and $\mathbf{Z}_{\mathbf{3}}$ in the direction of the axis of smallest inertia of Mercury. $h, g$ and $K$ are the Euler's angles linking both frames

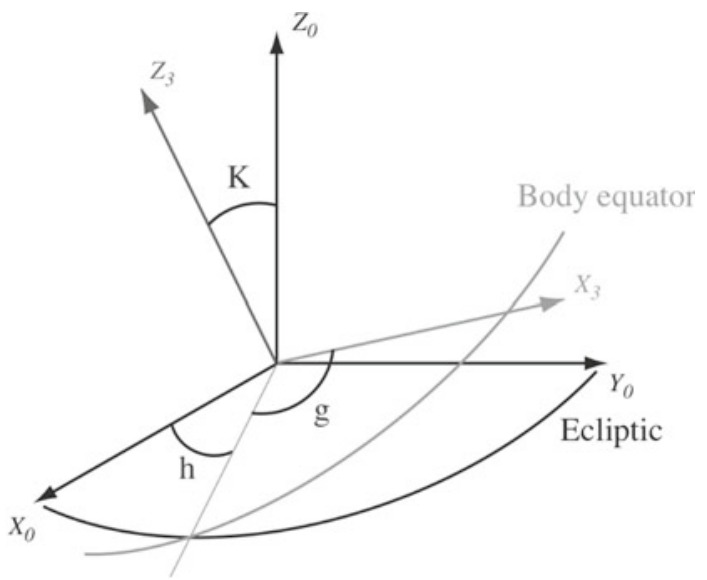

where $e$ the eccentricity, $i_{o}$ the orbital inclination, $m$ the mass of Mercury, $M$ the mass of the Sun, $R_{e}$ the equatorial radius of Mercury, $\mathcal{G}$ the gravitational constant, $\mu=\mathcal{G}(m+M), \Lambda_{o}$ the conjugated momentum of the mean anomaly $l_{o}$ and $C$ being the greatest moment of inertia.

In this Hamiltonian, the first term comes from the two-body problem, the second one is the kinetic energy of rotation and the third big one is the potential of the gravity field. The variables and their conjugated momenta are

$$
\begin{array}{ll}
\sigma_{1}=\frac{2(g+h)-3 l_{o}}{2}-h_{o}-g o & \Lambda_{1} \\
\sigma_{3}=-h+h_{o} & \Lambda_{3}=\Lambda_{1}(1-\cos K)
\end{array}
$$

where $g, h$ and $K$ are Euler's angles linking the body frame to the ecliptic frame (see Fig. 1), $h_{o}$ is the longitude of the ascending node, $g_{o}$ is the pericenter argument and $\Lambda_{1}$ is the angular momentum norm.

$\sigma_{1}$ is thus the 3:2 spin-orbit resonant angle and $K$ is called the ecliptic obliquity. The equilibrium values of this model found for the present state of Mercury were the following:

$$
\begin{aligned}
\sigma_{1} & =0, \\
\sigma_{3} & =0, \\
\Lambda_{1} & =13.303 \frac{m R_{e}^{2}}{\text { year }}, \\
K & =7^{\circ},
\end{aligned}
$$

and the proper periods of angles were:

$$
\begin{aligned}
& T_{1}=15.8573 \text { years }, \\
& T_{3}=1,065.08 \text { years. }
\end{aligned}
$$

\section{The Great Inequality of Jupiter and Saturn}

By introducing the perturbations due to the other planets of the Solar System on our kernel model, it appears that, in a particular case, our slow degree of freedom may enter in a 1:1 resonance with the Great Inequality of Jupiter and Saturn. This was already underlined by 
Dufey et al. (2009) who apply a perturbation theory based on the Lie triangle to re-introduce short periodic terms due to planetary perturbations into our averaged kernel Hamiltonian and so compute the evolution of the rotational variables (Dufey et al. 2008).

So, the period $T_{J S}$ of the Great Inequality of Jupiter and Saturn, obtained thanks to Simon's series, ${ }^{1}$ is

$$
T_{J S}=883.28 \text { years. }
$$

The proximity of the two periods $T_{3}$ and $T_{J S}$ led us to look for a perfect equality for a more realistic model of Mercury with a liquid core. Effectively, if we take a value of $C_{m} / C=0.82931$, this phenomenon may occur. However, according to (Margot et al. 2007), this value is not very probable but the parameters of Mercury are so poorly known that we must not exclude it (Figs. 5 and 6 in Dufey et al. 2009).

So, in this particular case, there is a 1:1 resonance between $\sigma_{3}$ and $\sigma_{25}=2 l_{J}-5 l_{S}$ where $l_{J}$ is the mean anomaly of Jupiter and $l_{S}$ the one of Saturn.

Let us thus introduce this angle $\sigma_{25}$ in the orbital elements thanks to Simon's series at first order:

$$
\begin{aligned}
e & =0.206+3.0304510^{-8} \cos \sigma_{25}+2.0541410^{-8} \sin \sigma_{25} \\
\cos i_{o} & =\cos 7^{o}+9.3790410^{-10} \cos \sigma_{25}+1.3366610^{-9} \sin \sigma_{25} \\
\sin i_{o} & =\sin 7^{o}-7.6386110^{-9} \cos \sigma_{25}-1.0886210^{-8} \sin \sigma_{25} \\
g_{o} & =29.12478^{o}+8.7453710^{-8} \cos \sigma_{25}-4.9462810^{-7} \sin \sigma_{25} \\
l_{o} & =l_{o_{0}}+5.7111110^{-8} \cos \sigma_{25}+5.1628110^{-7} \sin \sigma_{25} \\
h_{o} & =48.33^{o}+5.6200110^{-8} \cos \sigma_{25}+2.0722310^{-7} \sin \sigma_{25}
\end{aligned}
$$

where $l_{o_{0}}$ the mean anomaly without perturbations.

Our Hamiltonian can now be written:

$$
\left\langle\mathcal{H}_{25}\right\rangle=-\frac{m^{3} \mu^{2}}{2\left(\Lambda_{o}-\frac{3 \Lambda_{1}}{2}\right)^{2}}+\frac{\Lambda_{1}^{2}}{2 C_{m}}+V_{G}\left(\sigma_{1}, \sigma_{3}, \sigma_{25}, \Lambda_{1}, \Lambda_{3}\right)+v \Lambda_{25}
$$

with $v$ the frequency and $\Lambda_{25}$ the conjugated momentum of $\sigma_{25}$. Let us note that the last term has to be added in order to keep an autonomous Hamiltonian. The complete expression of $G$ is given in the Appendix.

\section{Secondary resonant angle}

In order to define our secondary resonant angle, we have to express our Hamiltonian in action-angle coordinates.

To do this, we have to perform a succession of transformations (D'Hoedt and Lemaître 2006):

- a canonical transformation into cartesian coordinates,

- a translation to the equilibrium,

- a Mac Laurin's expansion up to the order 2 for our first degree of freedom and up to the fourth order for our third degree of freedom,

${ }^{1}$ These series were given by J.-L. Simon in a private communication and are obtained from the VSOP theory (Fienga and Simon 2005). 
Fig. 2 During $\zeta$ performs a complete run, $\alpha$ oscillates between its minimum and maximum values

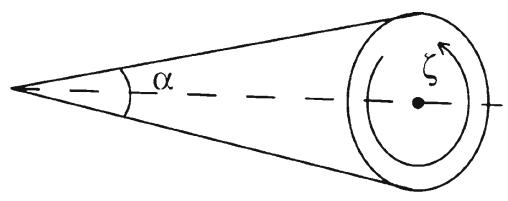

- an untangling transformation (Henrard and Lemaître 2005) to eliminate the mixed terms,

- a scale transformation to associate to each variable and its conjugate momentum the same coefficient,

- a transformation into action-angle coordinates $\left(J_{1}, J_{3}, \psi_{1}, \psi_{3}\right)$ where $\psi_{1}$ and $\psi_{3}$ are the angles of free libration around the equilibrium.

Let us note that we had to develop up to the fourth order to have terms in $J_{3}^{2}$.

We can now define our 1:1 secondary resonant angle

$$
\alpha=\psi_{3}-\sigma_{25} .
$$

Our canonical set of action-angle variables is thus now

$$
\begin{array}{ll}
\psi_{1} & J_{1} \\
\alpha & J_{3} \\
\sigma_{25} & \Lambda_{25}^{\prime}=J_{3}+\Lambda_{25} .
\end{array}
$$

The first degree of freedom $\left(J_{1}, \psi_{1}\right)$ being of no interest for the calculation of the period of our secondary resonant angle, we consider it as constant and drop it.

After that simplification, we average our Hamiltonian about $\sigma_{25}\left(\Lambda_{25}\right.$ becomes thus a constant) and obtain this expression:

$$
\begin{aligned}
\left\langle\mathcal{H}_{25}\right\rangle= & -0.000184 J_{3}^{2}+5.5047110^{-10} \sqrt{J_{3}} \cos \alpha \\
& -2.9766210^{-10} \sqrt{J_{3}} \sin \alpha .
\end{aligned}
$$

We again compute the equilibria of this Hamiltonian, perform a variables change to centre the Hamiltonian at the equilibrium and expand it in Mac Laurin's series up to the second order.

After a scaling transformation and an action-angle transformation, the final form of the Hamiltonian is

$$
\begin{aligned}
\left\langle\mathcal{H}_{25}\right\rangle= & -5.72710^{-8} Z+2.30710^{-5} Z^{2}-1.1510^{-6} Z^{3 / 2} \cos \zeta \\
& +1.1510^{-} 6 Z^{3 / 2} \cos 3 \zeta-2.30710^{-5} Z^{2} \cos 4 \zeta .
\end{aligned}
$$

The frequency of $\zeta$ being the coefficient of $Z$ in (14), we can deduce the period of $\zeta$ and thus the proper period of the free librations of $\alpha$ :

$$
T_{\alpha}=1.097210^{8} \text { years. }
$$

Actually, the period of $\zeta$ is also the one of the movement of $\alpha$ because during a complete run of $\zeta$ around its equilibrium, $\alpha$ performs one going and coming between its minimum and maximum values of libration (see Fig. 2).

\section{Influence of the secondary resonance on the other degrees of freedom}

If we compute and numerically integrate the equations of motion obtained from (11), we can see the influence of the secondary resonance on our principal degrees of freedom. 

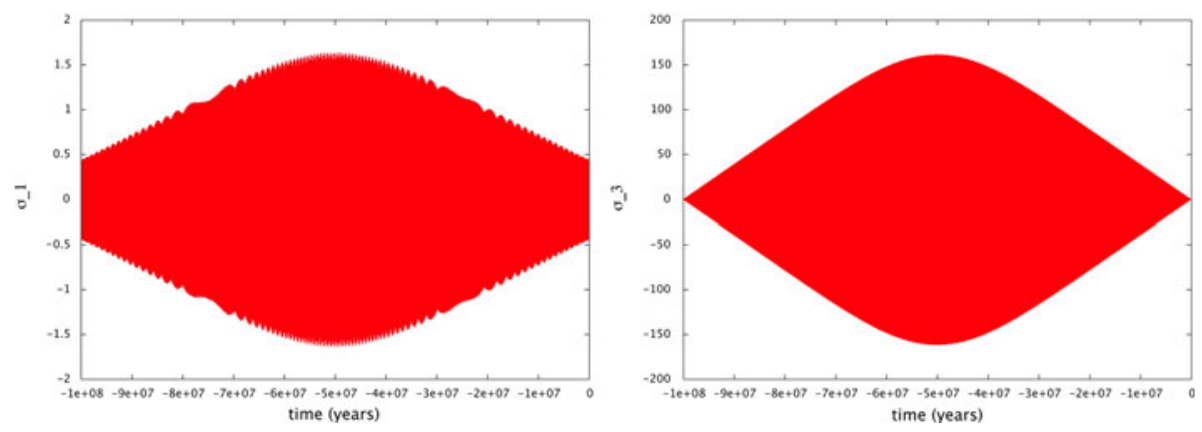

Fig. 3 Influence of the Great Inequality on $\sigma_{1}$ and $\sigma_{3}$

Fig. 4 Influence of the Great Inequality on the ecliptic obliquity $K$ (in degrees)

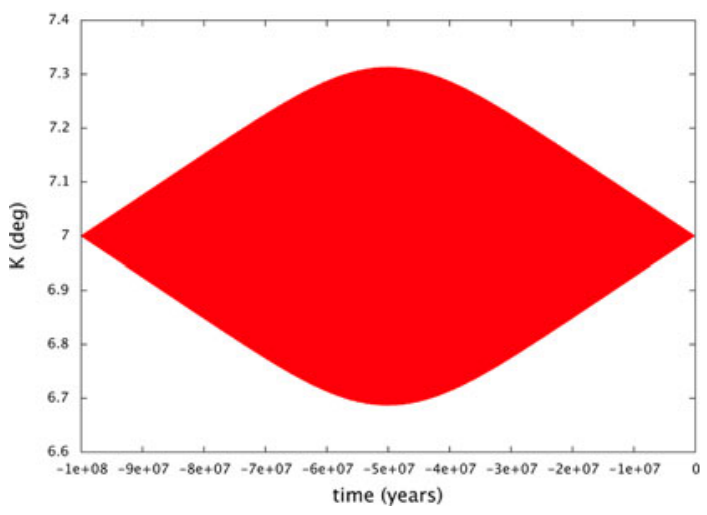

As expected, since the resonance is between $\sigma_{3}$ and the Great Inequality, its influence on $\sigma_{3}$ and $K$ is much bigger than on $\sigma_{1}$ : on $\sigma_{1}$, the amplitude is of $1.64 \operatorname{arcsec}$ (to be compared with a maximum amplitude of libration of $40 \mathrm{arcsec}$ Dufey et al. 2009), while it is of $161.49 \mathrm{arcsec}$ on $\sigma_{3}$ (Fig. 3) and of $0.3132^{\circ}=18.79 \mathrm{arc}$ min on the ecliptic obliquity $K$ (Fig. 4). This amplitude is especially large because if we examine the others effects that can introduce a forced libration on the ecliptic obliquity, we can see the most important of them is due to the precession of the ascending node of Mercury for which the amplitude of the variations are only of 0.414 arsec on a period of 63,315 years (Dufey et al. 2009). However, in both cases, the periods being very long, all what could be observed is constant offsets.

\section{Conclusion}

Due to the proximity of the period of one of our degree of freedom and of the Great Inequality of Jupiter and Saturn, we built a theoretical but nevertheless possible (with a weak probability) model taking into account this 1:1 secondary resonance. Even if the influence of this resonance on our main degrees of freedom is non negligible concerning the amplitudes, the proper period is so long that it could only be observed as a constant offset.

Acknowledgments The authors were supported by the contract Prodex C90253 ROMEO from BELSPO. The authors also would like to thank Jean-Louis Simon for kindly providing the planetary series. 


\section{Appendix: Complete expression of $V_{G}$}

$$
\begin{aligned}
& V_{G}=-\frac{\mathcal{G} m^{7} M R_{e}^{2} \mu^{3}}{\left(\Lambda_{0}-\frac{3 \Lambda_{1}}{2}\right)^{6}}\left[-\frac{3}{2} C_{2}^{0}\left(0.536+0.52 \cos ^{2} K+8.93910^{-9} \cos \sigma_{25}\right.\right. \\
& +1.06410^{-8} \cos ^{2} K \cos \sigma_{25}+0.004 \cos 2 \sigma_{3}-0.004 \cos ^{2} K \cos \sigma_{3} \\
& -4.25610^{-10} \cos \sigma_{25} \cos 2 \sigma_{3}+4.25610^{-10} \cos ^{2} K \cos \sigma_{25} \cos 2 \sigma_{3} \\
& +0.129 \cos K \cos \sigma_{3} \sin K-5.67710^{-9} \cos K \cos \sigma_{25} \cos \sigma_{3} \sin K \\
& +5.68910^{-9} \sin \sigma_{25}+8.32310^{-9} \cos ^{2} K \sin \sigma_{25} \\
& -6.58410^{-10} \cos 2 \sigma_{3} \sin \sigma_{25}+6.58410^{-10} \cos ^{2} K \cos 2 \sigma_{3} \sin \sigma_{25} \\
& -9.78410^{-9} \cos K \cos \sigma_{3} \sin K \sin \sigma_{25} \\
& +7.23110^{-9} \cos K \cos \sigma_{25} \sin K \sin \sigma_{3} \\
& -2.66610^{-8} \cos K \sin K \sin \sigma_{25} \sin \sigma_{3}+4.43910^{-10} \cos \sigma_{25} \sin 2 \sigma_{3} \\
& -4.43910^{-10} \cos ^{2} K \cos \sigma_{25} \sin 2 \sigma_{3}-1.63710^{-9} \sin \sigma_{25} \sin 2 \sigma_{3} \\
& \left.+1.63710^{-9} \cos ^{2} K \sin \sigma_{25} \sin 2 \sigma_{3}\right) \\
& +3 C_{2}^{2}\left(0.162 \cos \sigma_{1}+0.3245 \cos K \cos 2 \sigma_{1}\right. \\
& +0.162 \cos ^{2} K \cos 2 \sigma_{1}+1.91110^{-8} \cos 2 \sigma_{1} \cos \sigma_{25} \\
& +3.82310^{-8} \cos K \cos 2 \sigma_{1} \cos \sigma_{25}+1.91110^{-8} \cos ^{2} K \cos 2 \sigma_{1} \cos \sigma_{25} \\
& +0.004 \cos \left(2 \sigma_{1}+\sigma_{3}\right)-0.004 \cos ^{2} K \cos \left(2 \sigma_{1}+2 \sigma_{3}\right) \\
& +2.2710^{-6} \cos \left(2 \sigma_{1}+4 \sigma_{3}\right)-4.54110^{-6} \cos K \cos \left(2 \sigma_{1}+4 \sigma_{3}\right) \\
& +2.2710^{-6} \cos ^{2} K \cos \left(2 \sigma_{1}+4 \sigma_{3}\right)+0.04 \cos \left(2 \sigma_{1}+\sigma_{3}\right) \sin K \\
& +0.04 \cos K \cos \left(2 \sigma_{1}+\sigma_{3}\right) \sin K+2.16910^{-9} \cos \sigma_{25} \cos \left(2 \sigma_{1}+\sigma_{3}\right) \sin K \\
& +2.16910^{-9} \cos K \cos \sigma_{25} \cos \left(2 \sigma_{1}+\sigma_{3}\right) \sin K \\
& +0.0002 \cos \left(2 \sigma_{1}+3 \sigma_{3}\right) \sin K-0.0002 \cos K \cos \left(2 \sigma_{1}+3 \sigma_{3}\right) \sin K \\
& +3.79410^{-8} \cos \sigma_{25} \sin 2 \sigma_{1}+7.58710^{-8} \cos K \cos \sigma_{25} \sin 2 \sigma_{1} \\
& +3.79410^{-8} \cos ^{2} K \cos \sigma_{25} \sin 2 \sigma_{1}+1.30710^{-8} \cos 2 \sigma_{1} \sin \sigma_{25} \\
& +2.61410^{-8} \cos K \cos \left[2 \sigma_{1} \sin \sigma_{25}+1.30710^{-8} \cos ^{2} K \cos 2 \sigma_{1} \sin \sigma_{25}\right. \\
& -3.62110^{-10} \cos \left(2 \sigma_{1}+2 \sigma_{3}\right) \sin \sigma_{25} \\
& +3.62110^{-10} \cos ^{2} K \cos \left(2 \sigma_{1}+2 \sigma_{3}\right) \sin \sigma_{25} \\
& -3.74710^{-10} \cos \left(2 \sigma_{1}+\sigma_{3}\right) \sin K \sin \sigma_{25} \\
& -3.74710^{-10} \cos K \cos \left(2 \sigma_{1}+\sigma_{3}\right) \sin K \sin \sigma_{25} \\
& +1.5810^{-7} \sin 2 \sigma_{1} \sin \sigma_{25}+3.1610^{-7} \cos K \sin 2 \sigma_{1} \sin \sigma_{25} \\
& +1.5810^{-7} \cos ^{2} K \sin 2 \sigma_{1} \sin \sigma_{25}+1.15110^{-8} \cos \sigma_{25} \sin K \sin \left(2 \sigma_{1}+\sigma_{3}\right) \\
& +1.15110^{-8} \cos K \cos \sigma_{25} \sin K \sin \left(2 \sigma_{1}+\sigma_{3}\right) \\
& +3.04410^{-8} \sin K \sin \sigma_{25} \sin \left(2 \sigma_{1}+\sigma_{3}\right) \\
& +3.04410^{-8} \cos K \sin K \sin \sigma_{25} \sin \left(2 \sigma_{1}+\sigma_{3}\right) \\
& +1.26110^{-9} \cos \sigma_{25} \sin \left(2 \sigma_{1}+2 \sigma_{3}\right) \\
& -1.26110^{-9} \cos ^{2} K \cos \sigma_{25} \sin \left(2 \sigma_{1}+2 \sigma_{3}\right)+2.037710^{-9} \sin \sigma_{25} \sin \left(2 \sigma_{1}+2 \sigma_{3}\right) \\
& \left.\left.-2.03810^{-9} \cos ^{2} K \sin \sigma_{25} \sin \left(2 \sigma_{1}+2 \sigma_{3}\right)\right)\right]
\end{aligned}
$$




\section{References}

D’Hoedt, S., Lemaître, A.: The spin-orbit resonant rotation of Mercury: a two degree of freedom Hamiltonian model. Celest. Mech. Dyn. Astron. 89, 267-283 (2004)

D'Hoedt, S., Lemaître, A.: The spin-orbit resonance of Mercury: a Hamiltonian approach. Lect. Notes Phys. 682, 169 (2006)

D’Hoedt, S., Lemaître, A.: Planetary long periodic terms in Mercurys rotation: a two dimensional adiabatic approach. Celest. Mech. Dyn. Astron. 101, 127-139 (2008)

Dufey, J., Lemaître, A., Rambaux, N.: Planetary perturbations on Mercury's libration in longitude. Celest. Mech. Dyn. Astron. 101, 141-157 (2008)

Dufey, J., Noyelles, B., Rambaux, N., Lemaître, A.: Latitudinal librations of Mercury with a fluid core. Icarus 203, 1-12 (2009)

Fienga, A., Simon, J.-L.: Analytical and numerical studies of asteroid perturbations on Solar System planet dynamics. Astron. Astrophys. 429, 361-367 (2005)

Henrard, J., Lemaître, A.: Untangling transformation. Astron. J. 130, 2415-2417 (2005)

Margot, J.-L.: A Mercury orientation model including non-zero obliquity and librations. Celest. Mech. Dyn. Astron. 105, 329-336 (2009)

Margot, J.-L., Peale, S.J., Jurgens, R.F., Slade, M.A., Holin, I.V.: Large longitude libration of Mercury reveals a molten core. Science 316, 710-714 (2007)

Milani, A., Rossi, A., Vokrouhlický, D., Villani, D., Bonanno, C.: Gravity field and rotation state of Mercury from the BepiColombo radio science experiments. Planet. Space Sci. 49, 1579-1596 (2001)

Noyelles, B.: Titan's rotational state: the effects of a forced "free" resonant wobble. Celest. Mech. Dyn. Astron. 101, 13-30 (2008)

Peale, S.J.: Does Mercury have a molten core? Nature 262, 765-766 (1976)

Peale, S.J., Margot, J.-L., Phillips, R.J., Smith, D.E., Solomon, S.C., Zuber, M.T.: Interpretation of Mercury's rotation state. Bull. Am. Astron. Soc. 40, 490 (2008)

Peale, S.J., Margot, J.-L., Yseboodt, M.: Resonant forcing of Mercury's libration in longitude. Icarus 199, $1-8$ (2009) 